\title{
The Thermal Phase Sensitivity of both Coated and Uncoated Standard and Hollow Core Fibers down to Cryogenic Temperatures
}

\author{
W. Zhu, E.R. Numkam Fokoua, A.A. Taranta, Y. Chen, T. Bradley, M.N. Petrovich, F. Poletti, M. \\ Zhao, D.J. Richardson, and R. Slavík
}

\begin{abstract}
The thermal phase sensitivity of an optical fiber quantifies the degree to which a change in ambient temperature modifies the accumulated phase of light propagating through it. This sensitivity is often the limiting factor to the performance of fiber-based interferometers. Here we compare the thermal phase sensitivity of hollow core fiber (HCF) and standard single mode fiber (SMF-28) from $-180^{\circ} \mathrm{C}$ up to room temperature. We report measurements on fibers both with and without acrylate coating that enable an accurate estimation of the coating contribution.

The thermal phase sensitivity of fibers without any coating decreases at low temperatures. For SMF-28 it is reduced by a factor of four at $-190^{\circ} \mathrm{C}$ as compared to room temperature. For HCF, the thermal phase sensitivity becomes negative at low temperatures, crossing zero around $-70^{\circ} \mathrm{C}$, making the $\mathrm{HCF}$ operated at that temperature fully insensitive to small temperature fluctuations.

The coating significantly influences a fibers overall thermal phase sensitivity, especially at low temperatures, since it goes through a phase transition from a rubbery state at room temperature to stiff glassy state at low temperatures. We quantify the coating contribution and suggest fiber coating design rules to obtain fibers with reduced or even zero thermal phase sensitivity.
\end{abstract}

Index Terms-Optical fibers, Hollow core fiber, thermal sensitivities, Optical fiber interference.

\section{INTRODUCTION}

$\mathrm{T}$ HE thermally-induced optical phase change in fiber is detrimental in all fiber interferometers. For example, when using a fiber interferometer for frequency stabilization of a laser [1], [2] (for use in metrology [3] and sensing [4]), the thermal phase sensitivity of the fiber will convert temperature variations into frequency variations (a few $\mathrm{mK}$ of temperature change will result in hundreds of $\mathrm{kHz}$ frequency drift). Even in a vacuum environment, with active temperature stabilization, this effect represents the limiting factor in terms of performance of these

Manuscript received June xx, xxxx; revised August xx, xxxx; accepted xx xx. This work was supported by EPSRC project "Airguide Photonics", under grant EP/P030181/1, W. Zhu was supported by CSC scholarship, F. Poletti gratefully acknowledges EU ERC (grant 682724), R. Slavík was supported by RAEng Fellowship.

W. Zhu was with the Optoelectronics Research Centre, University of Southampton, Southampton SO17 1BJ, UK, and with the School of Optoelectronic Engineering and Instrumentation Science, Dalian University of Technology, Dalian 116024, China. systems [1]. In fiber optic gyroscopes, where the reciprocal path design of the Sagnac interferometer makes them mostly insensitive to small temperature fluctuations, the ultimate performance can still be limited by temperature changes via rapid thermal gradients (Shupe effect) [5].

The thermal phase sensitivity of a bare (uncoated) optical fiber comes from two effects [6]. The first is the refractive index change with temperature or thermo-optic effect $(1.1 \times$ $10^{-5} /{ }^{\circ} \mathrm{C}$ for fused silica glass); the second is linear thermal expansion which results in a small elongation of the fiber $(5 \times$ $10^{-7} /{ }^{\circ} \mathrm{C}$ in fused silica glass). For the case of fibers which are loosely wound and allowed to deform freely over thermal excursions, stress-optic effects resulting from thermal strains are negligible to first order. For a standard single mode fiber (SMF-28) at room temperature, the thermo-optic effect accounts for $95 \%\left(45 \mathrm{rad} / \mathrm{m} /{ }^{\circ} \mathrm{C}\right)$ of the total thermal phase sensitivity, while the thermally induced elongation only accounts for $5 \%\left(2.4 \mathrm{rad} / \mathrm{m} /{ }^{\circ} \mathrm{C}\right)[6]$. In hollow core fibers (HCF), such as the photonic bandgap fiber studied here, the thermooptic effect is virtually nil, as the light propagation is confined almost entirely in the thermally-insensitive air or vacuum-filled core [7]. This results in significantly lower thermal phase sensitivity $\left(\sim 2.4 \mathrm{rad} / \mathrm{m} /{ }^{\circ} \mathrm{C}[8]\right)$. To allow for easy comparison, the thermal phase sensitivity values in the article (including these from [6] and [8] shown above) are re-calculated following approach from [9] and normalized to the unit length.

It is interesting to consider operating fibers at low temperatures as a strategy to reduce or even eliminate the thermal phase sensitivity. Our preliminary results [9] show that bare SMF-28 has a four times lower thermal phase sensitivity at $-190^{\circ} \mathrm{C}$ (liquid nitrogen temperature) as compared to room temperature. At a significantly higher temperature $\left(-71^{\circ} \mathrm{C}\right)$, we have shown [11] that it is possible to achieve zero thermal phase sensitivity in a bare HCF. Below this temperature, the sign of the thermal phase sensitivity becomes negative, signifying that

E.R. Numkam Fokoua, A.A. Taranta, Y. Chen, T. Bradley, S.R. Sandoghchi, G.T. Jasion, M.N. Petrovich, F. Poletti, D.J. Richardson, and R. Slavík are with the Optoelectronics Research Centre, University of Southampton, Southampton SO17 1BJ, UK (R.Slavik@ soton.ac.uk).

M. Zhao is with the School of Optoelectronic Engineering and Instrumentation Science, Dalian University of Technology, Dalian 116024, China. 
a small temperature increase will result in less accumulated phase. The results from both SMF-28 and HCF suggest that in applications where the fiber thermal phase sensitivity is of importance, operation at low temperatures will furnish significant performance improvements.

All the fiber performance features we have discussed so far relate to bare fibers, which are fragile and notoriously difficult to handle. Thus, it is important to study the influence of the fiber coating on a fibers thermal phase sensitivity. At room temperature, this contribution is negligible in SMF-28 [12], but may need to be taken into account in HCFs, due to their intrinsically low thermal phase sensitivity. Dangui, et al [13] suggested reducing the coating contribution by making the silica glass cladding of the fiber as large as possible and using a thinner and softer coating material. For example, a HCF with a cladding diameter of $120 \mu \mathrm{m}$ and a coating diameter of 220 $\mu \mathrm{m}$ was reported to have a thermal phase sensitivity of 2.2 $\mathrm{ppm} /{ }^{\circ} \mathrm{C}$, corresponding to $8.5 \mathrm{rad} / \mathrm{m} /{ }^{\circ} \mathrm{C}$ [13]. Our in-house made $\mathrm{HCFs}$ with a significantly thicker silica cladding $(165 \mu \mathrm{m})$ and similar thickness of the coating showed thermal phase sensitivities as low as $2.4 \mathrm{rad} / \mathrm{m} /{ }^{\circ} \mathrm{C}$ at room temperature [8]. This is close to the value predicted from the thermal elongation of silica $\left(2.0 \mathrm{rad} / \mathrm{m} /{ }^{\circ} \mathrm{C}\right)$ [8] indicating that the coating contribution has been successfully reduced.

At low temperatures, however, the acrylate coating, which is soft at room temperature (with a Young's modulus three orders of magnitude below that of silica), goes through a glass transition from a rubbery state to a glassy state and becomes very stiff (with a Young's modulus comparable to silica glass). As the acrylate's coefficient of thermal expansion (CTE) is significantly higher than that of silica glass [14], its contribution to the fibers thermal properties at low temperatures (where it is very stiff) becomes significant [9].

In this paper, we present for the first time comprehensive measurements of the thermal phase sensitivities of SMF-28 and $\mathrm{HCF}$ from cryogenic to ambient temperatures in both coated and uncoated configurations. We quantify how the protective coating of the fiber contributes to the fiber's overall thermal phase sensitivity and use this to develop a framework for the design of low thermal phase sensitivity fibers. Using these tools we design a practical coated HCF with zero thermal phase sensitivity at low temperatures, thereby providing an alternative to the bulky and alignment-sensitive, ultra-low-expansion optical cavities used, for example, in metrology [15] and sensing.

\section{The Thermal Phase SENSITIVITY OF OPtICAL FIBERS}

\section{A. Bare optical fibers}

The thermal phase sensitivity $S_{\varphi}$ of light propagating through a unit length of an optical fiber is given by [9]:

$$
S_{\varphi}=\frac{1}{L} \frac{d \varphi}{d T}=\frac{2 \pi}{\lambda}\left(\frac{d n_{e f f}}{d T}+\alpha n_{e f f}\right)
$$

where $\lambda$ is the light wavelength in vacuum, $L$ is the fiber length, $T$ is the temperature, $\alpha$ is the effective CTE of the fiber, and $n_{\text {eff }}$ is the effective refractive index of the guided mode (typically 1.45 for SMF-28 and 0.997 for HCF [16]). The first term on the right-hand side is due to the thermo-optic effect, the second one is due to the thermally-induced fiber elongation. We can estimate $S_{\varphi}$ for SMF-28 by assuming the fiber's thermal properties are close to those of fused silica glass (which is a reasonable assumption as the fiber consists of a fused silica cladding and a lightly doped fused silica core). For a wide range of temperatures, the thermo-optic coefficient $(d n / d T)$ and CTE $(\alpha)$ of silica glass are given in [14] and [17], respectively. We can also calculate $S_{\varphi}$ for a $\mathrm{HCF}$ by assuming $d n / d T \approx 0$ for the air core which is a reasonable assumption for air at atmospheric pressure and a HCF with sealed-ends (such that no air escapes or enters the fiber) [18]. Expected values of $S_{\varphi}$ for uncoated fibers at a wavelength of $1550 \mathrm{~nm}$ (used throughout the entire paper) are in Fig. 1.

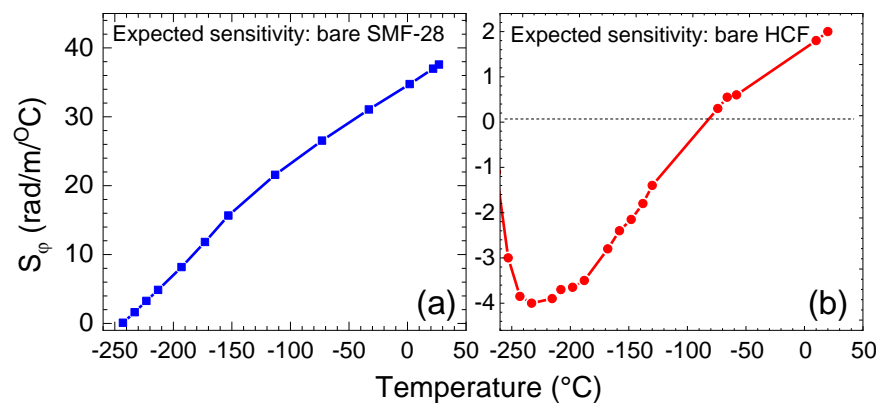

Fig. 1. The expected thermal phase sensitivities of bare SMF-28 (a) and bare HCF (b) based on the material properties of silica glass.

$S_{\varphi}$ is expected to change significantly for both forms of optical fiber as their temperature is decreased; for SMF-28 we expect $S_{\varphi}$ to reach 0 at $-250^{\circ} \mathrm{C}$, while for the $\mathrm{HCF}$, this occurs close to $-120^{\circ} \mathrm{C}$. Below these temperatures, $S_{\varphi}<0$, which presents an opportunity to compensate for the thermal expansion of the coating, as we will explain in subsequent sections.

\section{B. Coated optical fibers}

SMF-28 usually features two coating layers with the innermost (primary coating) having a glass transition temperature around $-50^{\circ} \mathrm{C}$ and the outer one (secondary coating) having a high glass transition temperature $\left(>80^{\circ} \mathrm{C}\right)$. In this paper, we use terminology used in the fiber manufacturing community, in which the primary and secondary coatings together are typically $62.5 \mu \mathrm{m}$ thick (for coated fibers with an outer diameter of $250 \mu \mathrm{m})$. Note that this terminology is somewhat different to that commonly used in the optical fiber communications community where these two coating layers are collectively referred to as the 'primary coating' and the term 'secondary coating' refers to any further protective layers of nylon or hytrel (e.g., forming a flexible $900 \mu \mathrm{m}$ thick over-cladded fiber).

For operation in the range of -50 to $+80^{\circ} \mathrm{C}$, the aforementioned properties yield a soft, compliant inner coating which cushions the glass fiber, and a hard outer coating which protects it from external stresses. Fig. 2 shows the key parameters of typical fiber coating materials (Young's modulus 
$E$ and $\alpha)$. Data given in the literature and by coating manufacturers generally provide the elastic modulus $E^{\prime}$, which is measured slightly differently to Yong's modulus. However, for our study, which deals with quasi-static changes (slow changes), we consider that $E \cong E^{\prime}$. The data shown in Fig. 2 for Young's modulus was taken from [19] (primary coating, -80 to $60^{\circ} \mathrm{C}$ ), from [20] (secondary coating, -20 to $60^{\circ} \mathrm{C}$ ), and from [21] (single-coating used on our in-house made HCF, -40 to $60^{\circ} \mathrm{C}$ ). Below temperatures for which data are available, all the considered coatings are in a glassy state in which Young's modulus usually changes very slowly with temperature. Considering this, we have extrapolated the available data to temperatures down to $-250^{\circ} \mathrm{C}$ to allow us to make qualitative predictions of the fibers' behavior. As concerns CTE, this increases linearly with temperature for common acrylate coating materials over a very large temperature range [22], [23], with a typical behavior [22], [23] shown in Fig. 2.

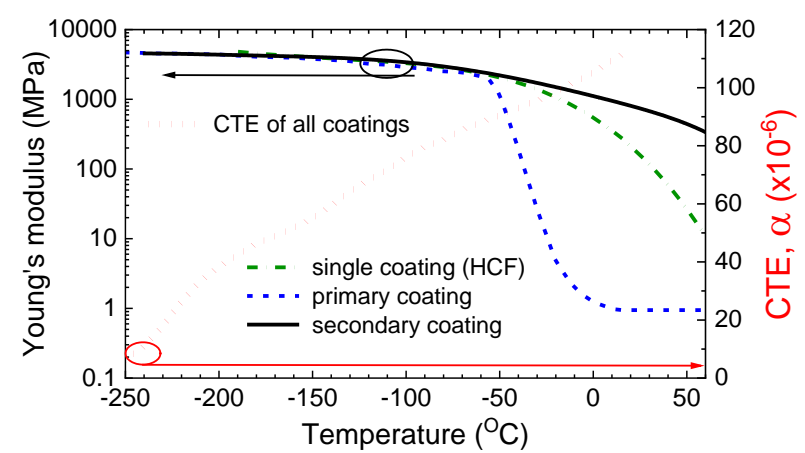

Fig. 2. The Young's modulus of acrylates we consider to be similar to those used as primary (blue, dashed) [19] and secondary (black, solid) [20] coatings of SMF-28 and single-coating of HCF (green, dash dot) [21]. Typical coefficient of thermal expansion $(\alpha)$ of acrylates is shown in red, dot [22], [23].

For the coated fiber, $S_{\varphi}$ depends on $\alpha$, the cross sectional area of the fiber $A$, and Young's modulus $E$ of all the materials used. For a fiber with a dual coating, we have three layers $(\mathrm{i}=1$ : silica glass fiber, $\mathrm{i}=2$ : primary coating, and $\mathrm{i}=3$ : secondary coating) and the effective longitudinal CTE of this composite can be calculated as follows [13], [23]:

$$
\alpha_{\text {coated fiber }}=\frac{\sum_{i=1}^{3} \alpha_{i} E_{i} A_{i}}{\sum_{i=1}^{3} E_{i} A_{i}}
$$

For SMF-28, we typically get glass fiber, primary and secondary coating diameters of $125 \mu \mathrm{m}, 190 \mu \mathrm{m}$, and $250 \mu \mathrm{m}$, respectively.

We calculated $S_{\varphi}$ of SMF-28 using Eqs. $(1,2)$, considering a constant silica glass Young's modulus of $72.5 \mathrm{GPa}$, with the rest of the parameters as discussed earlier. As can be seen from the result shown in Fig. 3, coated and bare SMF-28 have very different responses to temperature changes. We see an abrupt change around the primary coating glass transition temperature (around $-60^{\circ} \mathrm{C}$ ) with the maximum thermal phase sensitivity between $-60^{\circ} \mathrm{C}$ and $-100^{\circ} \mathrm{C}$. Within this temperature range the primary coating is already in the stiff glassy state (occurring below $-50^{\circ} \mathrm{C}$ ) and its thermal expansion coefficient is relatively high (as compared to even lower temperatures, Fig. 2). The thermal phase sensitivity of the fiber at $-250^{\circ} \mathrm{C}$ is about 5.6 $\mathrm{rad} / \mathrm{m} /{ }^{\circ} \mathrm{C}$, not zero as for the bare fiber, but still $>8$ times lower as compared to that at room temperature. The trend suggests the thermal phase sensitivity could be even further reduced at lower temperatures.

For HCF, we consider a 19-cell bandgap HCF designed with a core diameter of $29.2 \mu \mathrm{m}$, a microstructured cladding with a $90 \mu \mathrm{m}$ diameter, and with the surrounding silica jacket extending to $210 \mu \mathrm{m}$ in diameter. It has a single layer of acrylate coating (DeSolite 3471-3-14 [21]) of $50 \mu \mathrm{m}$ thickness with a Young's modulus as shown in Fig. 2. The calculated $S_{\varphi}$ is presented in Fig. 4, which also shows the bare HCF behavior for comparison. As with SMF-28, we see that the coating has a significant impact on the HCF's $S_{\varphi}$. This impact (in terms of how much it adds to $S_{\varphi}$ ), is smaller for HCF as compared to SMF-28 (Fig. 3), which can be understood from Eq. (2), especially at temperatures below $-50^{\circ} \mathrm{C}$, where all considered coating materials have similar Young's modulus (Fig. 2): the coating to cladding area ratio of SMF-28 is $\sim 3$, while it is only $\sim 1.4$ for the considered HCF.

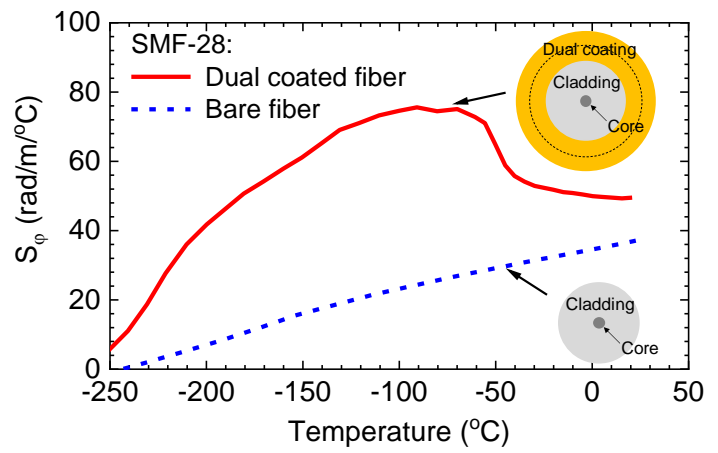

Fig. 3. The expected thermal phase sensitivity of dual coated SMF-28 (red, solid, $250 \mu \mathrm{m}$ outer diameter). Bare SMF-28 (from Fig. 1) is shown for comparison.

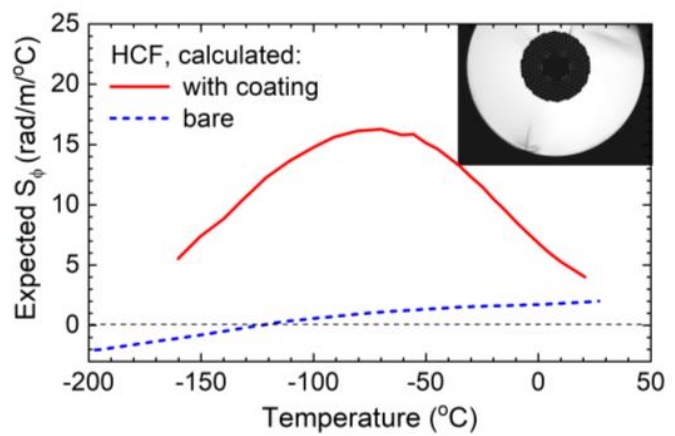

Fig. 4. The expected thermal phase sensitivity of the coated HCF with a glass diameter of $210 \mu \mathrm{m}$ and a coated diameter of $310 \mu \mathrm{m}$ (red, solid). Bare HCF (from Fig. 1) is shown for comparison. Inset: Photograph of the end face of the bare HCF.

\section{Thermal Phase SEnsitivity Measurement}

\section{A. Set-up}

To measure the optical phase change in a fiber under test (FUT), we placed it into the arm of a delay-line Mach-Zehnder interferometer (MZI), Fig 5. We carefully balanced the lengths 

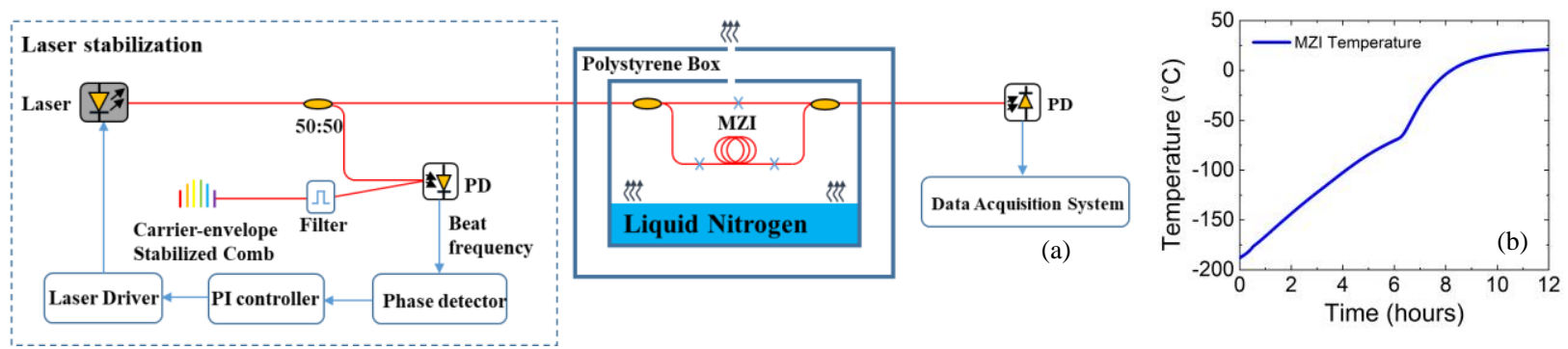

Fig. 5. (a) Experimental setup for measuring $S_{\varphi}$. Laser light with a frequency locked to an optical frequency comb passes through the MZI incorporating the FUT in one of its arms. As the $\mathrm{LN}_{2}$ evaporates, the temperature of the fiber rises (b) at an average rate of about $0.3^{\circ} \mathrm{C}$ per minute.

of the fiber coupler pigtails in both arms to minimize the impact of their thermal behavior during the experiment. We attached the MZI close to the top of a dual-walled polystyrene box.

For the testing of SMF-28 and coated HCF, the entire MZI apparatus was placed into a 20 -cm diameter glass Petri dish that was attached close to the top of a dual-walled polystyrene box. The box was filled with liquid nitrogen $\left(\mathrm{LN}_{2}\right)$ up to about $1 \mathrm{~cm}$ below the Petri dish and then closed. The $\mathrm{LN}_{2}$ evaporated slowly inside the closed box, lowering its level. As the $\mathrm{LN}_{2}$ level got lower, the temperature of the MZI rose, Fig. 5b. Two thermistors were placed on different sides of the MZI. Roughly 20 min after closing the box, the two thermistors started to show the same temperature of $-190^{\circ} \mathrm{C}$. From this point, the temperature rose at an average rate of $0.3^{\circ} \mathrm{C} / \mathrm{min}$, until reaching $-60{ }^{\circ} \mathrm{C}$ at which point the LN2 evaporated away.

Subsequently, the temperature increased more rapidly, but still slowly enough for the MZI to establish a uniform temperature distribution (confirmed by the reading from the two thermistors).

Note that in the case of bare HCF, which has an exceptionally low $\mathrm{S} \varphi$, we found that the residual contribution of the interferometer arms and couplers compromised our measurements when the whole MZI was placed in the $\mathrm{LN}_{2}$ filled box. Consequently, for these measurements, just the FUT was placed inside the box while the other MZI components (the couplers in particular) were moved outside of the box and maintained at a constant temperature (with active stabilization).

The total accumulated phase in each arm of the interferometer is $\varphi=2 \pi n_{e f f} L / \lambda$ and because the two MZI paths were balanced prior to insertion of the FUT (within $\sim 1$ $\mathrm{cm}$ ), any changes in differential phase between the two MZI arms could be attributed solely to the FUT. By simultaneously monitoring the temperature and the intensity at the output of the MZI, the phase offset accumulated by the FUT could then be mapped via the observed interferometric fringes. Over a given temperature excursion, the number of fringes that the output power slews across was used to calculate the thermal phase sensitivity according to:

$$
\mathrm{S}_{\varphi}=\frac{1}{L} \frac{d \varphi}{d T}=\frac{\text { number of fringes } \cdot 2 \pi}{\Delta T \cdot L}
$$

where $L$ is the FUT length, and $\Delta T$ is the temperature change over the observation time. To ensure that the observed changes were solely due to temperature drift and not wavelength drift of the light source, the laser (Rock laser from NP Photonics, operating at $1556 \mathrm{~nm}$ ) was locked to a carrier-envelopestabilized optical frequency comb (Menlo FC1500-250), Fig. 5 (a). After frequency stabilization, the light source output showed a long-term frequency accuracy better than $100 \mathrm{kHz}$, which was more than sufficient for our experiment in which the MZI spectral period was always $>10 \mathrm{MHz}$ (as determined by the relatively short FUT lengths).

\section{B. Results for $S M F-28$}

We measured uncoated (bare diameter $125 \mu \mathrm{m}$ ) and dualcoated ( $250 \mu \mathrm{m}$ diameter) SMF-28 samples. In addition to these two samples, we also tested a third sample having a $900 \mu \mathrm{m}$ 'tight buffer' jacket - a standard telecoms fiber configuration. We prepared three interferometers, with FUT lengths of $1.7 \mathrm{~m}$ $(125 \mu \mathrm{m}), 1.8 \mathrm{~m}(250 \mu \mathrm{m})$, and $1.1 \mathrm{~m}(900 \mu \mathrm{m})$, respectively. Fig. 6 shows the interference fringes at two different temperature ranges for bare SMF-28. We fitted the curve with a sinusoidal function to get an accurate period of each interference fringe (examples of typical fits shown in Fig. 6). We see that the interference fringes are sparser at lower temperatures as compared to higher temperatures, indicating that the bare SMF-28 has a lower $\mathrm{S}_{\varphi}$ at lower temperatures.

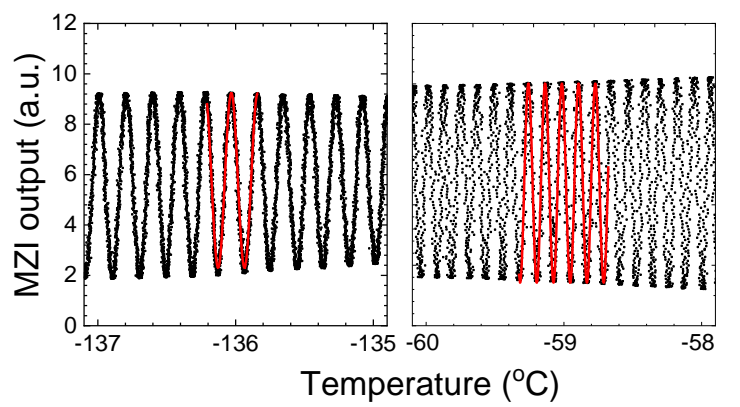

Fig. 6. Fringes output from bare SMF-28 based MZI at two temperatures ($136^{\circ} \mathrm{C}$ and $-59^{\circ} \mathrm{C}$ ) shown over the same temperature span (of $2^{\circ} \mathrm{C}$ ). Sinusoidal fits used to extract the fringe periods are shown as red solid lines.

Figure 7 shows the measured (and extracted using Eq. (3)) and expected (Fig. 1(a)) $\mathrm{S}_{\varphi}$ of the bare SMF-28, showing very good agreement, despite the fact that the silica fiber core is lightly doped with germanium. At $-186^{\circ} \mathrm{C}$, we measured $\mathrm{S}_{\varphi}=$ $10.9 \mathrm{rad} / \mathrm{m} /{ }^{\circ} \mathrm{C}$, which is almost four times lower than that at 
$20^{\circ} \mathrm{C}\left(37.5 \mathrm{rad} / \mathrm{m} /{ }^{\circ} \mathrm{C}\right)$. While the lower temperature bound of our experiment was limited by the boiling point of $\mathrm{LN}_{2}$, good agreement between the measured and modeled $\mathrm{S}_{\varphi}$ provides confidence in the model predictions, thus suggesting that this sensitivity can be further reduced, and will reach zero near $250^{\circ} \mathrm{C}$.

Subsequently, we measured $S_{\varphi}$ of the dual-coated and jacketed SMF-28, respectively, as shown in Figs. 8 and 9. For the dual-coated SMF-28, the measured values match reasonably well with the predicted values, especially considering that we do not know the exact composition and properties of the commercial SMF-28 coatings (which are not in public domain). $\mathrm{S}_{\varphi}$ increases from $48.5 \mathrm{rad} / \mathrm{m} /{ }^{\circ} \mathrm{C}$ (at $20^{\circ} \mathrm{C}$ ) to $73.2 \mathrm{rad} / \mathrm{m} / \mathrm{K}$ measured at $-80^{\circ} \mathrm{C}$. This increase is due to the primary acrylate coating undergoing a glass transition around $-50^{\circ} \mathrm{C}$. For even lower temperatures, the thermal phase sensitivity reduces, reaching $49.3 \mathrm{rad} / \mathrm{m} /{ }^{\circ} \mathrm{C}$ at $-190^{\circ} \mathrm{C}$, which is due to the reduction in the coating CTE.

For the 900- $\mu \mathrm{m}$ jacketed SMF-28, Fig. 9, the jacket contributes significantly more to the thermal phase sensitivity, e.g., at a temperature of around $-40^{\circ} \mathrm{C}$, the thermal phase sensitivity $\left(298 \mathrm{rad} / \mathrm{m} /{ }^{\circ} \mathrm{C}\right)$ is 10 times higher than that of the bare SMF-28. This shows that a thicker protective coating significantly increases the thermal phase sensitivity. This could be modelled by extending Eq. (2) into four layers, which we do not show here. The main point we would like to make is that the use of thick, standard coating materials is inadvisable for phasesensitive fiber applications operating at temperatures below $\sim 0^{\circ} \mathrm{C}$.

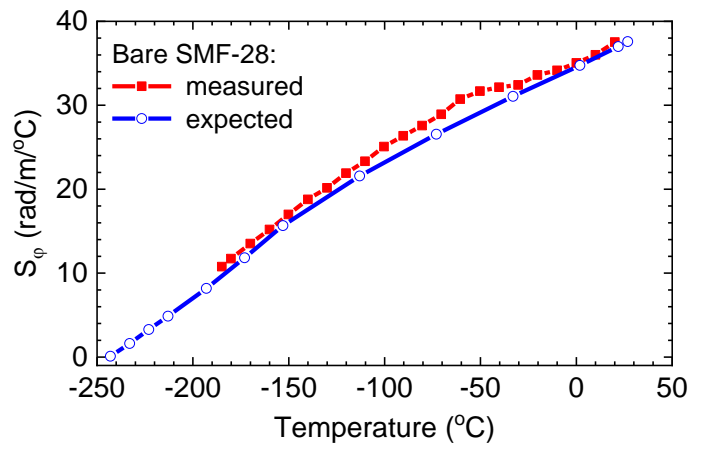

Fig. 7. Measured and expected thermal phase sensitivity of the bare SMF-28.

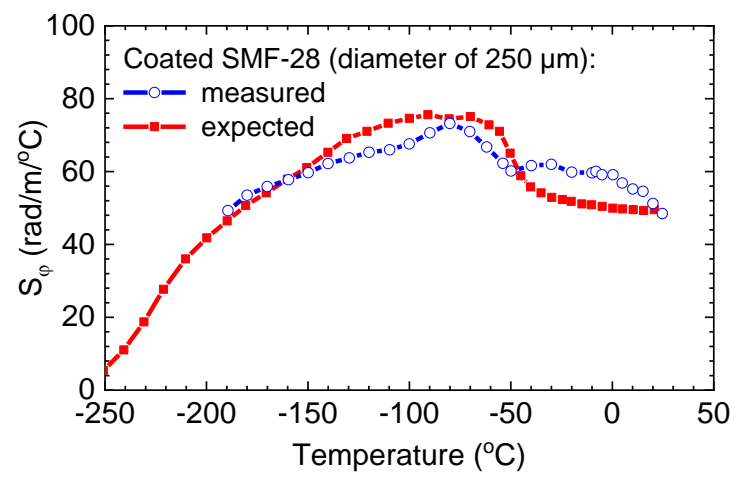

Fig. 8. Measured and expected thermal phase sensitivity of the dual-coated 250$\mu \mathrm{m}$ SMF-28 fiber.

\section{Hollow Core Fiber}

We tested a 19-cell photonic bandgap HCF manufactured inhouse and having key specifications of its microstructure as detailed in [8] and its dimensions specified in the above section. The FUT length was $8 \mathrm{~m}$ and $17 \mathrm{~m}$ for the bare and coated HCFs, respectively.

We have already published our results for bare HCF [8], and thus concentrate here on how this result allows us to isolate and analyze the coating contribution, and ultimately, how we could achieve zero thermal phase sensitivity with a practical protective coating. A detailed plot of the measured MZI interference fringes is shown in Fig. 10. As the temperature is increased, the spacing between fringes undergoes a marked change - from a very fine spacing at low temperature, to a very wide spacing near $-70^{\circ} \mathrm{C}$, and then back to a fine spacing at temperatures above that. We obtained $\mathrm{S}_{\varphi}$ using Eq. (3), as is shown in Fig. 11 (red solid line) together with the expected values (blue dashed line, from Fig. 1 (b)). The measured and expected curves match well from ambient temperatures down to $-110^{\circ} \mathrm{C}$. We believe the difference below $-110^{\circ} \mathrm{C}$ is due to changes in the thermal properties of the air inside the HCF [24]. The $\mathrm{S}_{\varphi}=0$ point was measured to occur at $-71^{\circ} \mathrm{C}$ with $\mathrm{S}_{\varphi}$ changing at a rate of $6 \times 10^{-9}{ }^{\circ} \mathrm{C}^{-2}$ around this point.

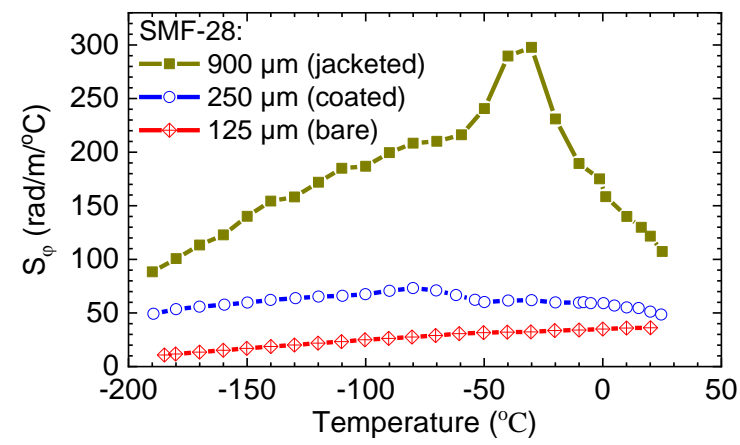

Fig. 9. Comparison of measured $\mathrm{S}_{\varphi}$ of SMF-28 that is bare (red cubic), 250$\mu \mathrm{m}$ dual-coated (blue circle), and $900-\mu \mathrm{m}$ jacketed (green square).

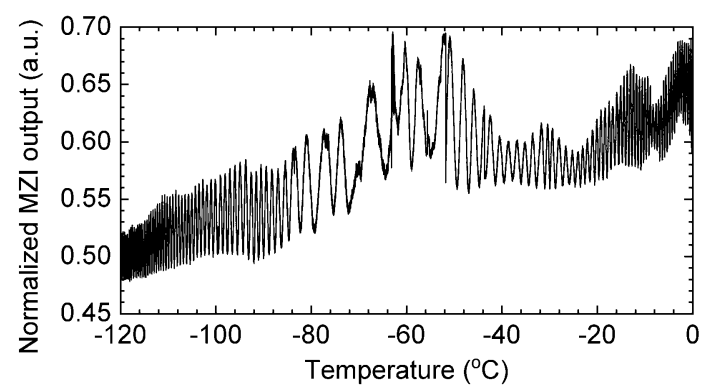

Fig. 10. Detail on the MZI interference fringes measured with the bare HCF FUT. 


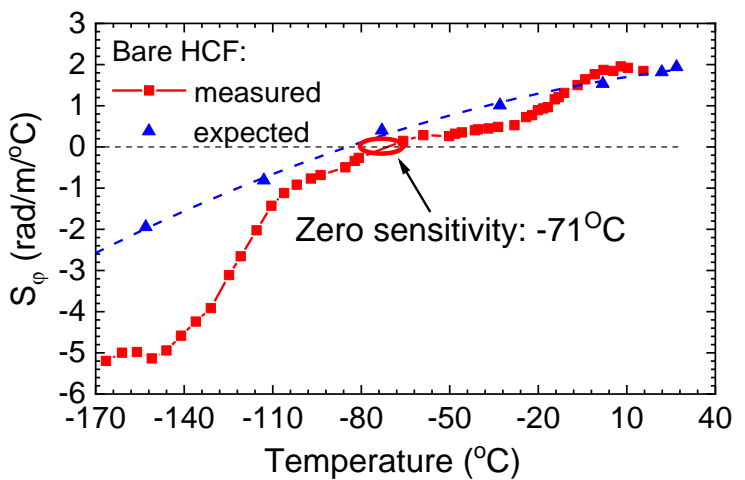

Fig. 11. Measured (red squares) and predicted (blue triangles, from Fig. 1) phase thermal sensitivity of the bare HCF.

Subsequently, we measured the coated HCF, Fig. 12. $\mathrm{S}_{\varphi}$ at room temperature was almost identical to that of the bare $\mathrm{HCF}$ (around $2 \mathrm{rad} / \mathrm{m} /{ }^{\circ} \mathrm{C}$ ). However, at low temperatures (below $\left.50^{\circ} \mathrm{C}\right) \mathrm{S}_{\varphi}$ became significantly higher, reaching 14-16 $\mathrm{rad} / \mathrm{m} /{ }^{\circ} \mathrm{C}$. Although this represents an increased phase sensitivity over the bare HCF, it is significantly smaller than that of coated SMF-28. At temperatures lower than $-120^{\circ} \mathrm{C}$, the measured $\mathrm{S}_{\varphi}$ does not follow the trend of the modeled curve. While we have not yet investigated this effect in detail, we hypothesize that it may arise due to changes in the thermal properties of the air inside the HCF [24], or unexpected changes in the acrylate material that were not captured by our extrapolation of its properties to low temperatures.

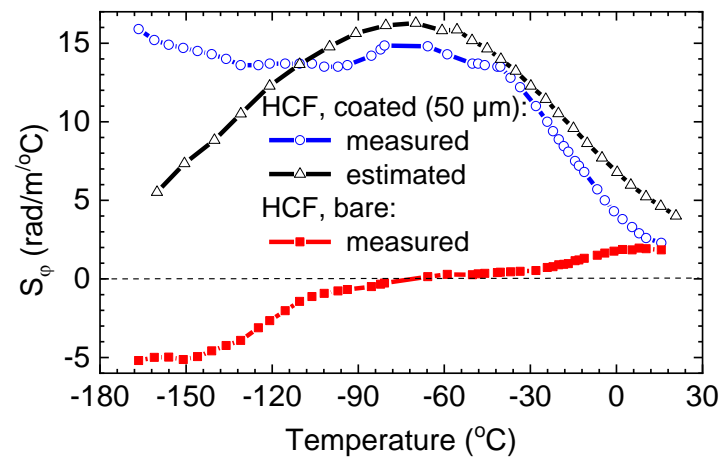

Fig. 12. Measured (blue circles) and predicted (black triangles) thermal phase sensitivity of a coated HCF with $50 \mu \mathrm{m}$ thick single coating. Bare HCF phase thermal sensitivity is shown for comparison (red squares).

\section{IMPROVING THERMAL PHASE SENSITIVITY OF COATED FIBERS}

Although we demonstrated significantly lower $\mathrm{S}_{\varphi}$ in bare fibers as compared to coated fibers, use of bare fibers is often impractical due to their fragility and associated handling challenges. Equation (2) suggests that reducing the coating thickness or increasing the area of the silica cladding will reduce $\mathrm{S}_{\varphi}$ of coated fibers, possibly to the point this can approach that of the bare fiber.

\section{A. Standard (solid core) fibers}

There are commercially available standard fibers with reduced coating thickness, e.g., using a thin polyimide coating. FiberCore (SM1500SC (7/125) [25]) and Nufern (NuSENSOR
$1550 \mathrm{~nm}$ Bend Insensitive Fiber [26]) have a standard cladding diameter of $125 \mu \mathrm{m}$ with the coated fiber being only $150 \mu \mathrm{m}$ in diameter. Such fiber is not fragile like its un-coated counterpart, but its insertion loss is generally slightly higher $(\sim 0.5 \mathrm{~dB} / \mathrm{km})$ than for $250-\mu \mathrm{m}$ coated SMF-28 $(<0.2 \mathrm{~dB} / \mathrm{km})$. This increase is due to fiber micro-bending induced loss, which is strongly suppressed in standard-coated SMF-28. However, we believe this slightly higher level of loss will be tolerable in most fiber interferometers (trading the thermal stability for the slight increase in loss). It is worth mentioning that there are other applications in which a thinner optical fiber is of interest - e.g., for datacenters, where it leads to improved space saving.

We simulated the performance of SMF-28 fiber incorporating a thin acrylate coating (thickness of $12.5 \mu \mathrm{m}$, giving a $150 \mu \mathrm{m}$ coated fiber diameter), Fig. 13 . We see that over the entire considered temperature range, $\mathrm{S}_{\varphi}$ of the thincoated fiber is only slightly higher than that of the bare SMF28. At $\mathrm{LN}_{2}$ temperature, the predicted thin-coated fiber $\mathrm{S}_{\varphi}$ is 13 $\mathrm{rad} / \mathrm{m} /{ }^{\circ} \mathrm{C}$, which is almost 4 times lower than that of the dualcoated $250-\mu \mathrm{m}$ thick SMF-28. At $-250^{\circ} \mathrm{C}$, the predicted $\mathrm{S}_{\varphi}$ is only $0.1 \mathrm{rad} / \mathrm{m} /{ }^{\circ} \mathrm{C}$ and may even reach zero slightly below this temperature.

\section{B. Hollow Core Fibers}

To calculate the $\mathrm{S}_{\varphi}$ of $\mathrm{HCFs}$ having different coating thickness (using Eq. (2)), we first applied Eq. (2) to calculate the coating CTE from the measured $\mathrm{S}_{\varphi}$ of the bare and $50-\mu \mathrm{m}$ thick coated HCFs, using the Young's modulus from Error! Reference source not found., see Fig. 2. Using coating CTE extracted in this way from our measurements (rather than using generic coating data shown in Fig. 2) should give more realistic prediction of the behavior of $\mathrm{HCFs}$ with thinner coatings.

Fig. 14 shows the predicted $\mathrm{S}_{\varphi}$ of the HCF with different thickness ( $35 \mu \mathrm{m}, 25 \mu \mathrm{m}$, and $12.5 \mu \mathrm{m}$, respectively). We see that in coated $\mathrm{HCF}, \mathrm{S}_{\varphi}$ can be reduced dramatically, especially when the coating is only $12.5 \mu \mathrm{m}$ (identical to the thickness of the commercial thin coated SMFs discussed earlier). Such thincoated HCF shows $\mathrm{S}_{\varphi}=0$ at $-130^{\circ} \mathrm{C}$.

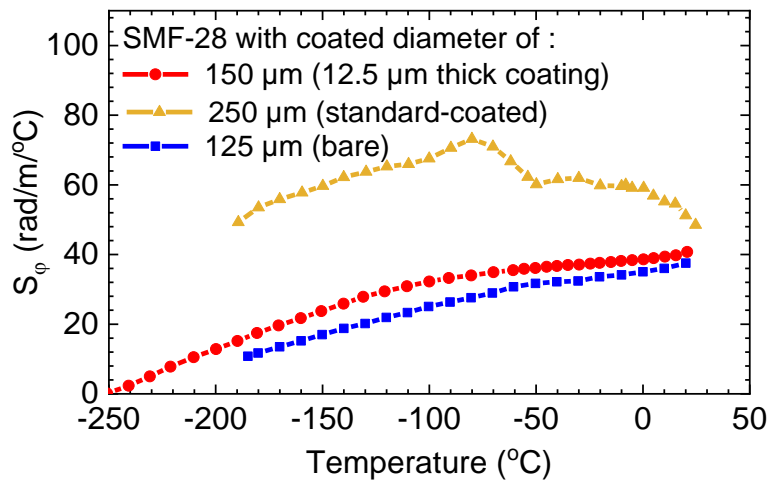

Fig. 13. Predicted thermal phase sensitivity of a thin-coated SMF-28 $(150 \mu \mathrm{m}$ outer diameter; coating thickness of $12.5 \mu \mathrm{m}$, red circles), together with the measured data for bare SMF-28 (125 $\mu \mathrm{m}$ diameter, blue squares) and coated SMF-28 (250 $\mu \mathrm{m}$ diameter, yellow triangles). 


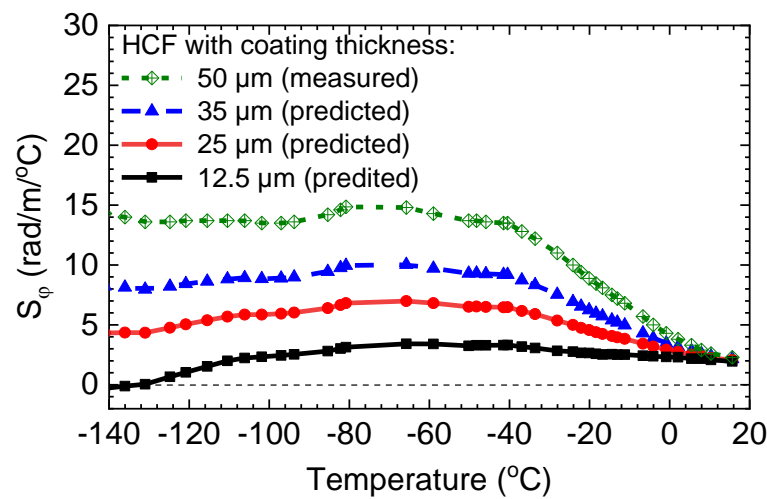

Fig. 14. Predicted thermal phase sensitivity of coated HCF with different coating thickness $(12.5-35 \mu \mathrm{m})$. For comparison, we also show the measured data for our HCF FUT (with a coating thickness of $50 \mu \mathrm{m}$ ) from which we extracted the properties of the coating (used to predict the HCF behavior with thinner coatings).

\section{COMPARISON}

Table 1 summarizes key phase sensitivity values for the studied fibers.

TABLE 1 Key parameters of fibers' phase sensitivity.

\begin{tabular}{|l|l|c|c|}
\hline \multirow{4}{*}{ Bare } & \multicolumn{1}{|c|}{$\mathrm{S}_{\varphi}$} & $\mathrm{SMF}$ & $\mathrm{HCF}$ \\
\cline { 2 - 4 } & Temperature at $\mathbf{S}_{\boldsymbol{\varphi}}=\mathbf{0}$ & $\mathbf{- 2 5 0} \mathbf{C}^{*}$ & $\mathbf{- 7 1}{ }^{\circ} \mathbf{C}$ \\
\cline { 2 - 4 } & $\mathrm{S}_{\varphi}, \mathrm{Temp}=20^{\circ} \mathrm{C}$ & $36 \mathrm{rad} / \mathrm{m} /{ }^{\circ} \mathrm{C}$ & $1.8 \mathrm{rad} / \mathrm{m} /{ }^{\circ} \mathrm{C}$ \\
\cline { 2 - 4 } & $\mathrm{S}_{\varphi}, \mathrm{Temp}=-100^{\circ} \mathrm{C}$ & $25 \mathrm{rad} / \mathrm{m} /{ }^{\circ} \mathrm{C}$ & $-0.6 \mathrm{rad} / \mathrm{m} /{ }^{\circ} \mathrm{C}$ \\
\hline \multirow{3}{*}{$\begin{array}{l}\text { Coated, } \\
\text { standard }\end{array}$} & Temperature at $\mathrm{S}_{\varphi}=0$ & - & - \\
\cline { 2 - 4 } & $\mathrm{S}_{\varphi}, \mathrm{Temp}=20^{\circ} \mathrm{C}$ & $45 \mathrm{rad} / \mathrm{m} /{ }^{\circ} \mathrm{C}$ & $2 \mathrm{rad} / \mathrm{m} /{ }^{\circ} \mathrm{C}$ \\
\cline { 2 - 4 } & $\mathrm{S}_{\varphi}, \mathrm{Temp}=-100^{\circ} \mathrm{C}$ & $65 \mathrm{rad} / \mathrm{m} /{ }^{\circ} \mathrm{C}$ & $12 \mathrm{rad} / \mathrm{m} /{ }^{\circ} \mathrm{C}$ \\
\hline \multirow{2}{*}{$\begin{array}{l}\text { Coated, } \\
\text { coating- } \\
\text { optimized }\end{array}$} & Temperature at $\mathbf{S}_{\varphi}=\mathbf{0}$ & - & $-\mathbf{- 1 3 5} \mathbf{C}^{*}$ \\
\cline { 2 - 4 } & $\mathrm{S}_{\varphi}, \mathrm{Temp}=20^{\circ} \mathrm{C}$ & - & $2 \mathrm{rad} / \mathrm{m} /{ }^{\circ} \mathrm{C}^{*}$ \\
\cline { 2 - 4 } & $\mathrm{S}_{\varphi}, \mathrm{Temp}=-100^{\circ} \mathrm{C}$ & - & $2 \mathrm{rad} / \mathrm{m} /{ }^{\circ} \mathrm{C}^{*}$ \\
\hline
\end{tabular}

* Predicted/calculated values

\section{CONCLUSION}

We predicted the phase thermal sensitivities $\mathrm{S}_{\varphi}$ of optical fibers (bare and coated SMF-28, and bare and coated HCF) from room temperature down to cryogenic temperatures. Subsequently, we carried out a comprehensive set of measurements from $\mathrm{LN}_{2}$ temperature up to room temperature, obtaining good agreement with our predictions.

When uncoated, both SMF-28 and HCF show markedly reduced $\mathrm{S}_{\varphi}$ at low temperatures as opposed to that at room temperature. The uncoated $\mathrm{HCF}$ reaches zero phase thermal sensitivity at $-71^{\circ} \mathrm{C}$ and exhibits negative values below this temperature. The sensitivity of the uncoated SMF-28, is almost 4 times lower at $\mathrm{LN}_{2}$ temperature $\left(-190^{\circ} \mathrm{C}\right)$ as compared to room temperature and it is predicted to reach zero around $250^{\circ} \mathrm{C}$.

Since bare fibers are impractical for handling, we also investigated the performance of fibers with protective coatings. Here we find that the coating degrades their $S_{\varphi}$ at low temperatures significantly. This is because typical acrylate coating materials that are in a soft rubbery state at room temperature go through a glass transition at low temperatures (around $-50^{\circ} \mathrm{C}$ ) and become very stiff. As these materials typically have a significantly larger coefficient of thermal expansion over that of silica glass, they then contribute significantly to the fiber elongation/contraction and thus to its thermal phase sensitivity.

With protective polymer coatings applied, the SMF-28 fiber showed an $\mathrm{S}_{\varphi}$ as high as $73 \mathrm{rad} / \mathrm{m} /{ }^{\circ} \mathrm{C}$ within the temperature range investigated (almost double that of the bare SMF-28 at room temperature). With an added jacketing layer $(900 \mu \mathrm{m})$, $\mathrm{S}_{\varphi}$ was as high as $300 \mathrm{rad} / \mathrm{m} /{ }^{\circ} \mathrm{C}$ (almost 8 times higher than for the bare SMF-28).

In our coated $\mathrm{HCF}$, the relative contribution from the coating is even more pronounced than for SMF-28, as bare HCFs are far less sensitive to temperature than SMF-28. The $S_{\varphi}$ was as high as $16 \mathrm{rad} / \mathrm{m} / \mathrm{K}$ over the studied temperature range, which is $>8$ times worse than for the bare $\mathrm{HCF}$ at room temperature.

To unlock the trade-off between practical handling (afforded by polymer coatings) and good performance (low sensitivity), we have also predicted the sensitivities of fibers with thinner coatings than those traditionally employed. With a very thin, yet achievable coating thickness of $12.5 \mu \mathrm{m}$, the $\mathrm{S}_{\varphi}$ of coated fibers could be reduced to levels approaching the performance of uncoated fibers. Indeed, with such a thin coating layer the predicted thermal phase sensitivity of SMF-28 at $\mathrm{LN}_{2}$ temperature is reduced by three times relative to that at room temperature, and is expected to reach zero at $-250^{\circ} \mathrm{C}$. These temperatures are today accessible using closed-loop Cryocoolers running from a standard electrical power socket. HCFs with such thin coatings are expected to achieve zero phase thermal sensitivity at $-130^{\circ} \mathrm{C}$. Such temperatures are accessible with only a Stirling engine, or a simple $\mathrm{LN}_{2}$ cooling system, making these techniques highly practicable for disciplines which require the most advanced, ultra-stable interferometers.

\section{ACKNOWLEDGMENT}

The data in this paper is accessible through the University of Southampton research repository (DOI: 10.5258/SOTON/XXXX).

\section{REFERENCES}

[1] K. Takahashi, M. Ando, and K. Tsubono, "Stabilization of laser intensity and frequency using optical fiber," in Journal of Physics: Conference Series, 2008.

[2] F. Kéfélian, H. Jiang, P. Lemonde, and G. Santarelli, "Ultralow-frequency-noise stabilization of a laser by locking to an optical fiber-delay line," Optics Letters, vol. 34, no. 7, pp. 914-916, 2009.

[3] J. Alnis, A. Matveev, N. Kolachevsky, T. Udem, and T. W. Hänsch, "Subhertz linewidth diode lasers by stabilization to vibrationally and thermally compensated ultralow-expansion glass Fabry-Pérot cavities," Physical Review A - Atomic, Molecular, and Optical Physics, vol. 77, no. 5, pp. 1-9, 2008.

[4] V. M. N. Passaro, A. Cuccovillo, L. Vaiani, M. De Carlo, and C. E. Campanella, "Gyroscope Technology and Applications: A Review in the Industrial Perspective," Sensors, vol. 17, no. 10, p. 2284, 2017. 
[5] D. M. Shupe, "Thermally induced nonreciprocity in the fiber-optic interferometer," Appllied Optics, vol. 19, no. 5, pp. 654-655, 1980.

[6] A. H. Hartog, A. J. Conduit, and D. N. Payne, "Variation of pulse delay with stress and temperature in jacketed and unjacketed optical fibres," Optical and Quantum Electronics, vol. 11, no. 3, pp. 265-273, 1979.

[7] F. Poletti et al., "Towards high-capacity fibre-optic communications at the speed of light in vacuum," Nature Photonics, vol. 7, no. 4, pp. 279-284, 2013.

[8] R. Slavík et al., "Ultralow thermal sensitivity of phase and propagation delay in hollow core optical fibres," Scientific Reports, vol. 5, pp. 1-7, 2015.

[9] S. Meiselman and G. A. Cranch, "Optical phase response to temperature in a hollow-core photonic crystal fiber," Optics Express, vol. 25, no. 22, p. 27581, 2017.

[10] W. Zhu, M. Ding, M. Zhao, D. J. Richardson, and R. Slavík, "The thermal sensitivity of optical path length in standard single mode fibers down to cryogenic temperatures," in Conference on Lasers and ElectroOptics (CLEO2019), 2019.

[11] W. Zhu et al., "Temperature insensitive fiber interferometery," Optics Letters, vol. 44, no. 11, 2019.

[12] M. Bousonville et al., "New phase stable optical fiber," in Proceedings of Beam Instrumentation Workshop, 2012, pp. 101-103.

[13] V. Dangui, H. K. Kim, M. J. F. Digonnet, and G. S. Kino, "Phase sensitivity to temperature of the fundamental mode in air-guiding photonic-bandgap fibers," Optics Express, vol. 13, no. 18, p. 6669, 2005.

[14] G. K. White, "Thermal expansion of reference materials: Copper, silica and silicon," Journal of Physics D: Applied Physics, vol. 6, no. 17, pp. 2070-2078, 1973.

[15] T. Kessler et al., "A sub-40-mHz-linewidth laser based on a silicon single-crystal optical cavity," Nature Photonics, vol. 6, no. 10, p. 687, 2012.

[16] F. Poletti, N. G. R. Broderick, D. J. Richardson, and T. M. Monro, "The effect of core asymmetries on the polarization properties of hollow core photonic bandgap fibers," Optics Express, vol. 13, no. 22, pp. 9115-9124, 2005.

[17] D. B. Leviton, B. J. Frey, N. Goddard, and S. Flight, "Temperature-dependent absolute refractive index measurements of synthetic fused silica," in Proceedings of SPIE, 2006.

[18] E. N. Fokoua, M. N. Petrovich, T. Bradley, F. Poletti, D. J. Richardson, and R. Slavík, "How to make the propagation time through an optical fiber fully insensitive to temperature variations," Optica, vol. 4, no. 6, pp. 659-668, 2017.

[19] S. R. Schmid and A. F. Toussaint, Specialty Optical Fibers Handbook, Chapter 4: Optical Fiber Coatings. pp. 95-122, 2007.

[20] DSM, "Product data for DeSolite ${ }^{\circledR}$ DS2042" [Online]. Available:

http://d7h5s3nxmpy4.cloudfront.net/CMP1007/files/Nex t-Gen-DeSolite-Supercoating-(Secondary-coatingDS2042)-Fiber-Optic-Center.pdf

[21] DSM, "Product data for DeSolite ${ }^{\circledR}$ 3471-3-14" [Online]. Available:
content/uploads/documents/AngstromBond---Fiber-OpticCenter-AngstromBond-DSM-3471-3-14-UV-Cure-FiberRe-Coating-(1Lt)-Fiber-Optic-Center.pdf.

[22] R. A. Haldon and R. Simha, "Thermal Expansivities of Polymers at Cryogenic Temperatures," Macromolecules, vol. 1, no. 4, pp. 340-343, 1968.

[23] M. Esposito et al., "Fiber Bragg Grating sensors to measure the coefficient of thermal expansion of polymers at cryogenic temperatures," Sensors \& Actuators: A. Physical, vol. 189, pp. 195-203, 2013.

[24] G. Bönsch and E. Potulski, "Measurement of the refractive index of air and comparison with modified Edlén's formulae," Metrologia, vol. 35, no. 2, pp. 133-139, 1998.

[25] FiberCore, "Pure Silica Core SM Fiber," 2019. [Online]. Available: https://www.fibercore.com.

[26] Nufern, "NuSENSOR 1550 nm Bend Insensitive SingleMode Fiber," 2016. [Online]. Available: https://www.nufern.com. 